\title{
Perspektiven ärztlicher Weiterbildung 2020
}

\author{
An einem Symposium am UniversitätsSpital Zürich erörterten Experten aus verschie- \\ densten Blickwinkeln folgende Fragen: Wohin soll sich die ärztliche Weiterbildung \\ entwickeln? Welche Tendenzen gibt es? Welche Anpassungen sind notwendig?
}

\author{
Hans Ulrich Buchera, \\ Jürg Hodler ${ }^{a}$, Werner Bauer ${ }^{b}$, \\ Christoph Hänggeli ${ }^{b}$ \\ a UniversitätsSpital Zürich \\ b Schweizerisches Institut für \\ ärztliche Weiter- und \\ Fortbildung SIWF
}

\section{Auslegeordnung}

Prof. J. Hodler, ärztlicher Direktor des Universitäts Spitals Zürich (USZ), führt aus, dass das USZ 621 Ärztinnen und Ärzte in Weiterbildung angestellt hat und die Spitaldirektion deshalb die ärztliche Weiterbildung als Kernthema bezeichnet.

Dr. W. Bauer ist seit 5 Jahren Präsident des SIWF. Dieses ist das Kompetenzzentrum der Schweiz rund um die ärztliche Weiter- und Fortbildung. Es begleitet als selbständiges Institut, fachlich unabhängig von der FMH und akkreditiert vom EDI/BAG, alle diplomierten Ärztinnen und Ärzte in der Schweiz während ihrer beruflichen Laufbahn nach dem Staatsexamen [1].

C. Hänggeli, Rechtsanwalt und Geschäftsführer des SIWF, erläutert, dass sich zurzeit über 6000 Ärztinnen und Ärzte in Weiterbildung befinden und dass im Jahr 20131645 Weiterbildungstitel vergeben worden sind. Es gibt 45 anerkannte Weiterbildungstitel und dazu noch eine grosse, zunehmende Anzahl Schwerpunkte und Fähigkeitsausweise. Viele der Weiterbildungsprogramme wurden angepasst oder sind noch in Revision. Wichtig dabei ist, dass die allgemeinen Lernziele wie medizinische Ethik, Medizinökonomie, Management generell gefordert werden und dass mindestens einmal ein Wechsel der Weiterbildungsstätte erfolgen soll. Ab dem 1.7.2015 ist beim Einreichen der Unterlagen für den Titel die Dokumentation der Weiterbildung mit einem elektronischen Logbuch obligatorisch.

Dank der Visitationen und der jährlichen Evaluationen durch die Leiter der Weiterbildungsstätten und die Weiterzubildenden wissen wir, dass für die überwiegende Mehrheit die Weiterbildung die notwendigen Kompetenzen vermittelt, das Verhältnis Dienstleistung zu Lernen adäquat ist und sich praktisch alle qualifizierten stationären und ambulanten Institutionen an der Weiterbildung beteiligen.

Problemfelder sind, dass Titel und Curricula nicht «marketingorientiert» sein sollen und dass die Finanzierung der Weiterbildung durch die Kantone noch umstritten ist.

\section{Innovation}

A. Greulich, Leiter des Medizinbereichs Herz-GefässThorax am USZ, stellt seinen Bereich als neue Organisationsform vor, die mehrere Kliniken integriert.
Diese haben eine grosse Autonomie, überlassen jedoch gewisse organisatorische Aufgaben wie die Zuteilung von Operationssälen und Patientenbetten dem Bereich. Innerhalb des Medizinbereichs können Weiterbildungscurricula, die sich auf dasselbe Organ beziehen wie z. B. Pneumologie und Thoraxchirurgie, besser aufeinander abgestimmt werden.

Prof. N. Schäfer, Oberarzt an der Klinik für Nuklearmedizin USZ, erläutert, wie sich aus der Nuklearmedizin die Theragnostik entwickelt hat, d.h. wie eine spezifische Bildgebung zu spezifischen Interventionen führt. Damit rückt sie näher an die Neurologie, Onkologie, Radiologie und sogar die Psychiatrie, was Auswirkungen auf die Weiterbildung in allen diesen Disziplinen hat.

Prof. P. A. Clavien, Direktor der Klinik für Viszeralund Transplantationschirurgie, stellt eine zunehmende Spezialisierung fest, die sich auf die ärztliche Weiterbildung auswirkt. Einerseits besteht ein Druck für die Etablierung neuer Fachrichtungen und zur Zentralisierung, andererseits wird es immer schwieriger, Generalisten auszubilden. Wissenschaftliche Untersuchungen zeigen, dass die Qualität der Patientenversorgung und der Weiterbildung mit der Anzahl der Eingriffe pro Institution und pro Chirurg zunimmt [2]. Eine Umsetzung dieser Erkenntnisse wird erschwert durch den Wunsch der Bevölkerung nach regionalen Spitälern mit kurzen Anfahrtswegen und der Begrenzung der Arbeitszeiten für Assistenz- und Oberärzte.

Prof. M. Thali, Direktor des Instituts für Rechtsmedizin UZH, zeigt auf, wie die Rechtsmedizin in den letzten Jahren mit der rasanten Entwicklung der Virtopsie ihren Fokus völlig geändert hat. Damit überschneidet sie sich stark mit der klinischen Radiologie. Diese Entwicklung ist im aktuellen Weiterbildungscurriculum für die Rechtsmedizin noch nicht berücksichtigt, eventuell wird daraus ein neuer Titel oder mindestens ein neuer Schwerpunkt.

\section{Entwicklung des Arbeitsmarktes}

PD Dr. A. Zinkernagel, Leitende Ärztin an der Klinik für Infektionskrankheiten und Spitalhygiene USZ, berichtet von ihrer Erfahrung als Ärztin, Wissenschaftlerin und Mutter. Sie weist darauf hin, dass heute die Mehrheit der Ärzte in Weiterbildung Frauen sind. Um diese zu motivieren, in Klinik oder 


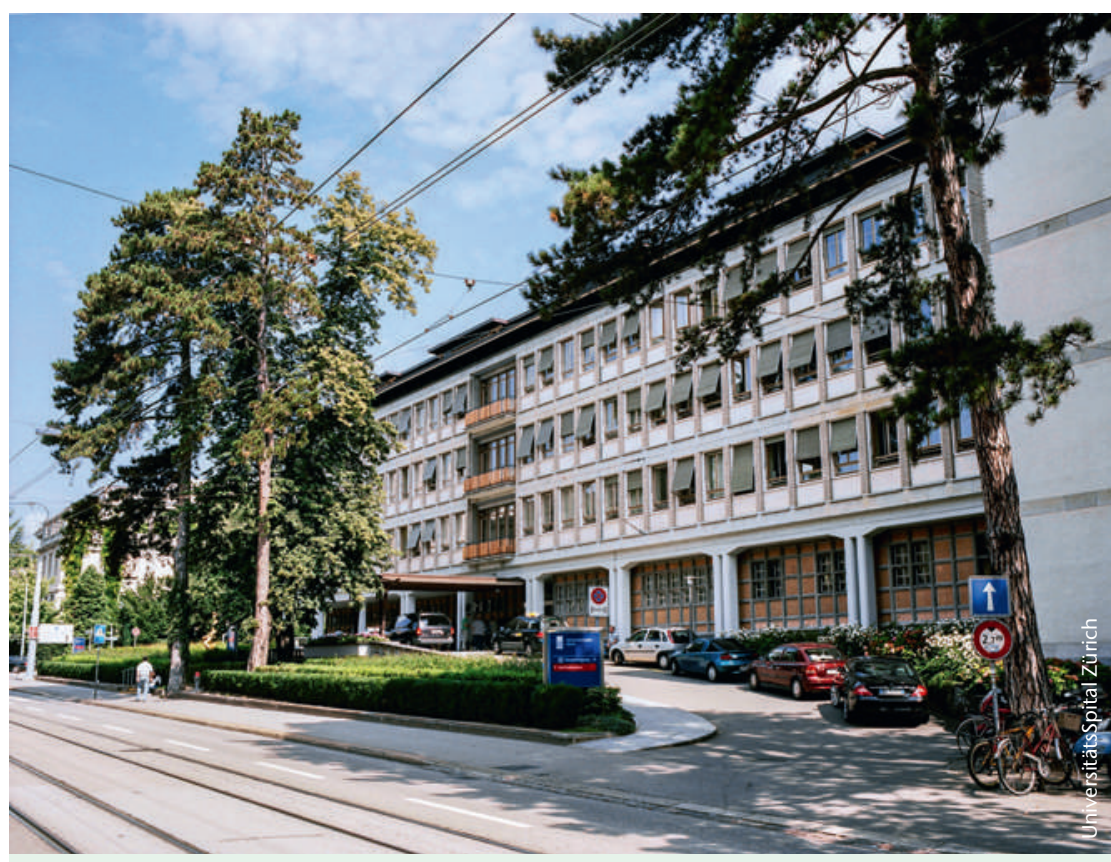

Das UniversitätsSpital Zürich will die Wichtigkeit der Weiterbildung betonen und hat hierzu einen Weiterbildungsbeauftragten benannt.
Forschung Karriere zu machen, braucht es Veränderungen in der Weiterbildung. Dazu gehören flexible Arbeitszeiten, Kindertagesstätten, die über die üblichen Bürozeiten hinaus geöffnet sind, und vor allem Vorbilder und ein gutes Coaching. Eine besondere Förderung benötigen Frauen, die sich neben ihrer klinischen Tätigkeit in der Forschung profilieren wollen. Dazu wären die in den USA bewährten «Clinical fast tracks» geeignet und «Tenure tracks», die nach einer strengen Selektion eine Anstellung garantieren [3].

Dr. Ch. Peter, stv. Medizinischer Leiter von mediX Zürich, zeigt auf, dass Grosspraxen rasch wachsen und offenbar einem Bedürfnis sowohl von Patienten als auch von Ärzten und vor allem Ärztinnen entsprechen. Für diese sind Teilzeitarbeit (mindestens 50\%) und Leistungslohn (basierend auf TARMED), Delegation vieler administrativer Aufgaben und der wirtschaftlichen Verantwortung sowie die Sicherheit durch laufende Besprechungsmöglichkeit mit Kollegen attraktive Punkte.

Prof. G. Hofbauer, Leitender Arzt an der Dermatologischen Klinik USZ, berichtet über seine Erfahrungen mit Weiterbildungsstellen auf Aussenstationen. Dort können wertvolle praktische Erfahrungen mit banalen medizinischen und administrativen Problemen gesammelt werden. Diese Stellen sind allerdings nicht sehr beliebt, da sie regelmässig besetzt werden müssen, unter Umständen längere Arbeitswege in Kauf genommen werden müssen und die
Supervision und damit die Weiterbildungsqualität recht unterschiedlich sind.

\section{Fazit und Ausblick}

Prof. J. Hodler bekräftigt, dass die ärztliche Weiterbildung für das USZ ein Kerngeschäft ist und bleibt. Aus diesem Grund hat die Spitaldirektion einen Weiterbildungsbeauftragten institutionalisiert. Diese neue Funktion wird ab März 2014 durch Prof. H. U. Bucher, ehemaliger Klinikdirektor, in Teilzeitanstellung wahrgenommen. Die Weiterbildner sollten grundsätzlich mehr Anerkennung und Unterstützung erhalten. Ein Schritt in diese Richtung ist die Projektausschreibung des SIWF.

Eine Reihe von Problemen sind noch zu lösen: Eine adäquate Finanzierung der Weiterbildung muss sichergestellt werden. Die Weiterbildung muss so angepasst werden, dass eine Kombination von Klinik, Forschung und Familie attraktiv wird. Dazu gehören kurze Wege zwischen Klinik, Labor und Kindertagesstätte. Hidden Agendas von Fachgesellschaften müssen verhindert werden. Die Kluft zwischen Einhaltung der Arbeitszeiten und der geforderten minimalen Zahl von Eingriffen muss überwunden werden.

Dr. W. Bauer nennt als besondere Herausforderungen für die ärztliche Weiterbildung neben der Finanzierung deren Steuerung gemäss dem Bedarf des Gesundheitswesens, die sinnvolle Definition von Lernzielen und die Anwendung sinnvoller Evaluationsmethoden zur Qualitätsüberprüfung. Er betont den Nutzen von Weiterbildungs-Netzwerken. Er ist kritisch gegenüber den universitären CAS («Certificate of advanced studies»), die in Konkurrenz zu den Fähigkeits- und Fertigkeitsausweisen stehen. Eine weitere Verkürzung der Weiterbildungsdauer hält er für unrealistisch, ist diese doch in den letzten Jahren schon deutlich zurückgegangen. Es gilt, die treibenden Kräfte im Gesundheitswesen wie technische und pharmakologische Innovation, Markt, Geldgeber und Politik im Auge zu behalten und die ärztliche Weiterbildung ständig anzupassen. Bei der anhaltenden Reform der ärztlichen Weiterbildung sollten wir die Aussage von William Osler nicht vergessen: «We expect too much of the student and we try to teach him too much. Give him good methods and a proper point of view, and all other things will be added, as his experience grows.»

\section{Referenzen}

1 Bauer W, Hänggeli C. Kleines Jubiläum - grosse Aufgaben. Schweiz Ärztezeitung. 2014;95(15):605.

2 Hutter MM. Specialization: the answer or the problem? Ann Surg. 2009;249:717-8.

3 Andrews NC. The other physician-scientist problem: Where have all the young girls gone? Nature Medicine. 2002;8: 439-41. 